\section{Feasibility, efficacy and safety of tyrosine kinase inhibitor treatment in hemodialyzed patients with renal cell cancer: 10 years of experience}

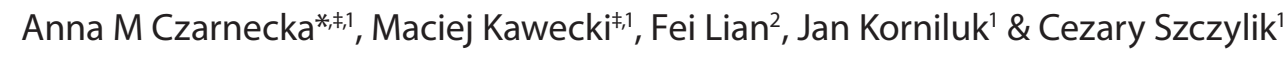

\begin{abstract}
Aims: Sine efficiency of tyrosine kinase inhibitor (TKI) therapy in dialyzed patients is still unclear we aim to analyze the outcome of treatment in such cohort. Patients \& methods: We analyzed treatment outcomes of patients with clear cell renal cell carcinoma (ccRCC) with special focus on those who were also treated with hemodialysis and described treatment safety and progression-free survival of eight patients treated with TKIs and hemodialysis. Discussion \& conclusion: Our report supports statement that TKI treatment of dialyzed patients is safe and effective. ccRCC increases risk of developing renal insufficiency as well as end-stage renal disease that require dialysis. Introduction of multitargeted receptor kinase inhibitors (TKIs), including sunitinib, sorafenib and pazopanib significantly expanded life time expectancy of metastatic renal clear cell carcinoma. The advance also applies to patients with ccRCC and end-stage renal disease who undergo dialyses.
\end{abstract}

Targeted therapies are currently considered the standard of care for metastatic renal cell carcinoma (mRCC) patients. Five VEGF pathway inhibitors including tyrosine kinase inhibitors (TKIs) - sorafenib, sunitinib, pazopanib and axitinib; monoclonal antibody directed against VEGF bevacizumab (in combination with IFN- $\alpha$ ); and mTOR inhibitors (mTORIs) - everolimus and temsirolimus are now US FDA approved for mRCC. This compounds have been registered based on Phase III randomized clinical trials data [1-5]. Several subgroups of patients, including hemodialyzed patients, are routinely excluded from enrollment in clinical trial due to strict eligibility criteria. Pivotal Phase III trials excluded patients with creatinine concentration $\geq 1.5$ the upper limit of norm and expanded access trail with $>2.0$. As a consequence the toxicity and efficacy of targeted therapies in populations with chronic kidney disease and end-stage renal disease (ESRD) are largely unknown [6]. Currently also little information is available on TKIs dosage or schedule adjustment in patients with ESRD. Exclusion of ESRD patients form large trials precludes a considerable number of patients from accessing of potentially effective targeted therapies in trials and after FDA/EMA approval on one hand. On the other hand it also hinders the collection of adequate pharmacokinetic, safety and efficacy data on this specific patient population that could facilitate approval of drugs in case of concurrent ESRD and RCC [7].

One may expect cases of suffering from $\mathrm{mRCC}$ - and potentially eligible for TKI treatment among long-term dialyzed patients. It was shown that maintenance of renal function was shown to plays a critical role in chemotherapy and may possibly be so in case of targeted therapies in mRCC. It was shown that if renal excretion is significantly reduced, lower drug elimination may occur and as a result the risk of toxicity may increase - relatively to increased concentration of medication in serum/blood [8]. Other ESRD-related changes that can alter the pharmacokinetics of a drug

'Department of Oncology, Military Institute of Medicine, Warsaw, Poland

2Emory University School of Medicine Atlanta, GA, USA

*Author for correspondence: Tel.: +48 226817 172; Fax: +48 226103 098; anna.czarnecka@gmail.com

${ }^{\ddagger}$ Authors contributed equally

\section{KEYWORDS}

- end-stage renal disease

- hemodialysis - pazopanib

- renal cell cancer • renal insufficiency $\bullet$ sorafenib - sunitinib 
include hypoalbuminemia, presence of edema, metabolic acidosis, abnormal enteric drug metabolism or reabsorption [7]. As a result of all these factors unexpected adverse events (AEs) may be observed or known AEs may be amplified in intensity or duration. On the contrary a decreased pharmacological anticancer effect may also occur [7]. In routine clinical practice physicians are therefore confronted with the question of whether patients with terminal renal failure can be safely treated for mRCC [9]. Therefore, treatment of patients with mRCC and concurrent ESRD still represents a challenge for medical oncologist [10]. Limited literature data on such treatment is currently available, despite its clinical relevance. Currently there are no summaries or recommendations for TKIs in terms of dialysis and above mentioned indices. Patients with ESRD are often excluded from prospective Phase III clinical trials because of their expected altered pharmacokinetics, comorbidities and life expectancy [11]. Limited data are available regarding patients with RCC and hemodialized ESRD treated with TKIs. The objectives of our study was to investigate the safety and efficacy of TKIs in hemodialized patients with mRCC. We aim to address lack of ESRD-TKI treatment data through our analysis of data from the Oncology Department in the Military Medicine Institute in Warsaw combined with data gained from comprehensive literature analysis.

\section{Patients \& methods}

With objective to investigate the incidence, safety and efficacy of TKI treatment in patients with metastatic RCC and ESRD requiring hemodialysis (HD) 679 consecutive patients with $\mathrm{mRCC}$ were screened in the Military Institute of Medicine (MIM). Screened cohort included patients 18 years of age or older with confirmed RCC who were treated in MIM in Warsaw, starting the treatment between March 2004 and March 2014. Patient inclusion criteria were as reported before, including confirmed RCC, good or intermediate performance status (ECOG 0-2), low- or intermediate-risk Memorial Sloan-Kettering Cancer Center (MSKCC) score and proir nephrectomy or NSS [2,4,12-15]. In this cohort patients eligible for the treatment were treated with TKI as per clinical practice and all patients gave written informed consent according to institutional MIM requirements. The patients started the treatment with: sunitinib $50 \mathrm{mg}$ orally once daily in 6-week cycles according to a 4-week on/2-week off treatment schedule; or sorafenib in $400 \mathrm{mg}$ twice daily schedule; or pazopanib $800 \mathrm{mg}$ once daily $[13,16-17]$. The patients were monitored as reported before. Toxicities, including renal parameters, were monitored as per standard treatment protocol [1,18-19].

Baseline evaluation (Table 1) in current analysis include patients renal function, duration of ESRD, mode and duration of dialysis (including type of dialysis), data regarding nephrectomy status, previous therapies, TKI drug dosage, tumor stage and grade, histological subtype of tumor, information on AEs, progression-free survival (PFS), last available MIM follow-up and date of death (all causes). Adverse events were graded according to the Common Terminology Criteria for Adverse Events, version 3.0 and 4.0 (since May 2009).

Time (duration) of treatment (TOT) was defined as the time elapsed between the initiation of TKI treatment (TT) and the discontinuation of that TT for any reason. PFS was defined as the time between the initiation of TT and the discontinuation of that TT due to progression defined by RECIST criteria or death. Response was accessed by the treating providers with Response Evaluation Criteria In Solid Tumors (RECIST 1.0). Baseline patient characteristics and toxicity data were described with summary statistics. Patient characteristics were summarized using medians and ranges for continuous variables and frequency and percentage for categorical variables. Survival curves were estimated using the Kaplan-Meier method.

Literature data review covered PubMed/ MEDLINE/ASCO Meeting library data on sunitinib, sorafenib, pazopanib or tyrosine kinase inhibitor and renal insufficiency, renal impairment or hemodialysis published 2000-2014.

\section{Results}

Out of 679 patients, 464 patients were treated with TKI (68\%); among those nine patients (1.3 and $1.9 \%$, respectively) subjects were treated with dialysis due to ESRD. Patients baseline characteristics are summarized in Table 1. Among those cases clear cell renal cell carcinoma (ccRCC) TKI treatment was conducted as follows: five patients were treated with sunitinib, three with sorafenib, one with pazopanib. Moreover, two patients were treated with HD were treated with INF - as were ineligible for TKI, and one patient with HD did not receive 
treatment due to poor performance status and rapid progression. After first-line treatment two patients received second-line therapy. The estimated median PFS of this cohort was 8 months and mean PFS was 8.5 months (95\% CI: 4.901-12.099). Partial response was achieved in one patient, five patients presented with stable disease as best response and progressive disease was found in two patients (Table 2). With regard to safety and tolerability most AEs were grade 1 or 2 and grade $5 \mathrm{AEs}$ were not reported. No significant interruptions to treatment with TKI were reported in patients examined in this study. Dose adjustment consisted of decreasing the dose in one patient. In this patient lower dosed was introduced after temporal 4-week treatment interruption due to AEs (thrombocytopenia). One patient discontinued treatment due to grade 4 hypertension, although this patient has hypertension prior to TKI Therapy. There was no significant differences in outcome in patient who was treated on reduced dose and those on full dose. The time between the start of HD and the treatment with TKI (time-delay dialysis therapy) was less than 12 months. In one patient, sorafenib-sunitinib (TKI + TKI) treatment was conducted while other patient was treated primarily with interferon and was later switched to sorafenib.

Patient 1 was a 54-year-old male patient with Alport Syndrome on dialysis three times a week since December 2010. A routine ultrasonography scan performed in February 2011 detected presence of a left kidney tumor and patient nephrectomy that revealed ccRCC. In August 2011, metastases in lungs, liver and peritoneum were found. With Eastern Cooperative Oncology Group (ECOG) 1 and intermediate prognosis according to MSKCC model started treatment with sunitinib at a dose of $50 \mathrm{mg}$ daily $4 / 2$. During therapy, patient suffered from mild adverse effects: grade 2 hand-foot syndrome and leukopenia, grade 1 hypertension and diarrhea. Patient 2 was a 65 -year-old male who patient underwent nephrectomy due to ccRCC tumor in horseshoe kidney in September 2012. He was later was hemodialyzed since nephrectomy. In April 2013 this patient was diagnosed with numerous metastases in lungs and regional recurrences. This patient was treated with sunitinib $50 \mathrm{mg} \mathrm{4/2}$ at ECOG 1 and with a poor prognosis in MSKCC. Early treatment tolerance was acceptable as patient developed only grade 2 diarrhea, but in August 2013 patients'
Table 1. Baseline of Military Institute of Medicine patients receiving tyrosine kinase inhibitors and hemodialysis.

\begin{tabular}{ll} 
Baseline patient characteristics $(\mathbf{n}=\mathbf{8})$ & $\mathbf{n}(\%)$ \\
Median age (range), years & $65(34-70)$ \\
Sex: & \\
- Female & $1(12.5)$ \\
- Male & $7(87.5)$ \\
Histology: & \\
- Clear cell & $6(75)$ \\
- Papillary & $2(25)$ \\
Previous nephrectomy & $7(87.5)$ \\
Type of dialysis: & \\
- Hemodialysis & $7(87.5)$ \\
- Peritoneal dialysis & $1(12.5)$ \\
Time of dialysis introduction: & \\
- After diagnosis & $5(62.5)$ \\
- Less than 1 year prior to diagnosis & $3(37.5)$ \\
- More than 1 year prior to diagnosis & $0(0)$ \\
Prognostic feature: & \\
- KPS score <80 & $0(0)$ \\
- Time from diagnosis to treatment <1 year & $5(62.5)$ \\
- Hemoglobin <LLN & $5(62.5)$ \\
- Corrected calcium >10 mg/dl & $2(25)$ \\
- LDH >1.5-fold the ULN & $1(12.5)$ \\
MSKCC risk subgroup: & \\
- Favorable & $2(25)$ \\
- Intermediate & $4(50)$ \\
- Poor & $2(25)$ \\
Sites of metastases: & \\
- Lung & $6(75)$ \\
- Liver & $6(75)$ \\
- Lymph nodes & $5(62.5)$ \\
- Bones & $4(50)$ \\
- Other & $4(50)$ \\
Local recurrence of the disease & $4(50)$ \\
\hline KPS: Karnofsky performance status; LDH: Lactate dehydrogenase; LLN: Lower limit of normal; \\
MSKCC: Memorial Sloan-Kettering Cancer Center; ULN: Upper limits of normal.
\end{tabular}

performance status deteriorated and grade 3 anemia and fatigue developed. Patient 3 was a 33-year-old female patient, with a previous history of a metastatic Wilms' tumor treated successfully with a right side nephrectomy and a subsequent radiochemotherapy at the age of 3 years, who developed left kidney tumor and underwent nephrectomy for papillary RCC. Due to removal of only kidney, patient started hemodialysis, while 3 months after surgery metastases were found in lungs, pancreas, liver and bones. With intermediate MSKCC prognosis in November 2007 the patient started sorafenib at a dose of $800 \mathrm{mg}$ daily. In September 2008 after progression was switched to sunitinib $50 \mathrm{mg}$ 4/2. After skeletal PD in November 2009 


\begin{tabular}{|c|c|c|c|c|c|c|c|}
\hline Patient & Start & Stop & $\begin{array}{l}\text { PFS } \\
\text { (months) }\end{array}$ & Dose/24 h & Drug & $\begin{array}{l}\text { Best } \\
\text { response }\end{array}$ & Toxicity \\
\hline 1 & $\begin{array}{l}\text { August } \\
2011\end{array}$ & $\begin{array}{l}\text { December } \\
2012\end{array}$ & 16 & $50 \mathrm{mg}$ & SU & SD & $\begin{array}{l}\text { Grade } 2 \text { hand-foot syndrome and leukopenia, } \\
\text { grade } 1 \text { hypertension and diarrhea }\end{array}$ \\
\hline 2 & May 2013 & $\begin{array}{l}\text { August } \\
2013\end{array}$ & 3 & $50 \mathrm{mg}$ & SU & PD & Grade 2 diarrhea \\
\hline 3 (first line) & $\begin{array}{l}\text { November } \\
2007\end{array}$ & $\begin{array}{l}\text { September } \\
2008\end{array}$ & 10 & $800 \mathrm{mg}$ & SOR & SD & Tolerated well \\
\hline $\begin{array}{l}3 \text { (second } \\
\text { line) }\end{array}$ & $\begin{array}{l}\text { September } \\
2008\end{array}$ & $\begin{array}{l}\text { November } \\
2009\end{array}$ & 14 & $50 \mathrm{mg}$ & SU & SD & Tolerated well \\
\hline 3 (third line) & $\begin{array}{l}\text { February } \\
2010\end{array}$ & May 2010 & 3 & $10 \mathrm{mg}$ & EVE & PD & Tolerated well \\
\hline 4 & May 2007 & $\begin{array}{l}\text { September } \\
2007\end{array}$ & 4 & $400-800 \mathrm{mg}$ & SOR & PR & Tolerated well \\
\hline 5 & May 2012 & July 2012 & 2 & $50 \mathrm{mg}$ & SOR & SD & Bleeding from rectal varices and fatigue grade $2 / 3$ \\
\hline 6 & July 2009 & March 2010 & 8 & $800 \mathrm{mg}$ & SOR & SD & Grade 4 hypertension \\
\hline 7 & $\begin{array}{l}\text { November } \\
2009\end{array}$ & July 2010 & 8 & $37.5 \mathrm{mg}$ & SU & SD & Thrombocytopenia (31,000 plates/ $\mu \mathrm{l})$ \\
\hline 8 & April 2011 & $\begin{array}{l}\text { August } \\
2011\end{array}$ & 4 & $800 \mathrm{mg}$ & PAZ & PD & Grade 2 fatigue and hand-foot syndrome \\
\hline
\end{tabular}

radiotherapy and subsequent everolimus treatment were used. Patient 4 was a 47 -year-old male patient with left side renal agenesis and right kidney ccRCC diagnosed in July 2003. Three months after nephrectomy due to local recurrence patient was treated with interferon (IFN; 3 million units per dose) and achieved complete remission (CR). After 23 months (June 2005) due local recurrence patient underwent subsequent surgery and in November 2005 lung metastases were treated with IFN with SD. In May 2007 due to liver, spleen and soft tissues metastases patient started sorafenib $2 \times 200 \mathrm{mg}$ daily that was increased to $2 \times 400 \mathrm{mg}$ daily after first cycle. Patient 5 was 65 -year-old male patient with polycystic kidney disease, who underwent right-sided nephrectomy in April 2011 for ccRCC, developed metastatic disease in March 2012 and CKD in May 2012 in the month sunitinib $50 \mathrm{mg} \mathrm{4/2}$ was started. Since patient suffered from Type 2 diabetes, obesity and hypertension, and reported bleeding from rectal varices and fatigue in June 2012 dose was reduced to $37.5 \mathrm{mg}$ daily. Patient 6 was a 67-year-old male with a previous history of a right-side nephrectomy due to hydronephrosis on dialysis since April 2009. In May 2009 left nephrectomy was performed due to papillary RCC type I. Patients' comorbidities included hypertension, atrial fibrillation, congestive heart failure and Type 2 diabetes. In July 2009 patient started sorafenib $2 \times 400 \mathrm{mg}$ daily due to metastases in lungs, lymph nodes and liver. Early treatment tolerance was good, but after three cycles patient developed grade 4 hypertension and treatment was discontinued. Patient 7 was a 69-year-old male patient with a history of a prior right-side nephrecotmy in 1991 and was diagnosed with left kidney ccRCC in September 2008. In October 2009 due to local recurrence and metastases in liver, vertebral column, and in dorsal muscles radiotherapy was conducted and in November $200950 \mathrm{mg}$ sunitinib was initiated. After 2 weeks of treatment patients developed thrombocytopenia $(31,000$ plates $/ \mu \mathrm{l})$ and sunitinib was temporally withdrawn. After normalization of parameters sunitinib was reintroduced in reduced dose of $37.5 \mathrm{mg}$ daily. Treatment tolerance was acceptable, with toxicities limited to I grade hand-foot syndrome and grade II mucositis. In July 2010 patients' status deteriorated, as he developed asthenia, fatigue, hematoma in left groin and a bleed to right oculi and CT scan revealed PD. Finally, patient 8 was a 63 -year-old male patient with ischemic heart disease and hypertension and renal failure due to renal fibrosis of a right kidney and a large ccRCC tumor in a left kidney in October 2010. Synchronically metastases in lungs, lymph nodes and pelvis were diagnosed. Starting April 2011 patient was treatment with pazopanib $2 \times 400 \mathrm{mg}$ for four cycles until PD. 
Discussion

- TKI treatment efficacy in hemodialyzed patients

A sizeable number of RCC patients with RCC have renal impairment have been reported in prior publications. Until today total number of 61 patients with renal insufficiency treated with hemodialysis and sunitinib (Table 3) and 29 with sorafenib (Table 4) until now [9,20-28]. We report on additional eight patients treated with TKI and HD. Median PFS in our patients was 8 months, while in the pivotal trial first-line sunitinib treatment PFS was 11 months and second-line (after IFN/IL-2) sorafenib - 5.5 months [1,2]. In the expanded access trial median PFS for sunitinib treatment was 10.9 months and for sorafenib from 6.6 [15] to 9 months [29]. Our treatment results were within the expected range of survival, also if compared with data for HD treated patients - who had median PFS of 12.7 months and mean PFS 11.55 (95\% CI: 9.38-13.72) months and median PFS of 13.0 months and mean PFS 13.09 (95\% CI: 7.89-18.29) in sorafenib cohort (Tables 3 \& 4). Nevertheless, the direct comparison is not possible at this point of time since data reported before (Tables 3 \& 4) include usage of TKIs in various lines of therapy, both after and without cytokine treatment. The limitation of our study is also the unavailability of second/third-line of treatment - including mTOR inhibitor due to reimbursement regulation of National Health Found. Moreover, lots of data in previous reports must be considered as censored as PFS is reported as longer than particular number of months, but total PFS is not presented.

Together with our reported patients presented above, we analyzed a cohort of patients treated with sunitinib and HD (Table 3). Majority of the patients were treated with hemodialysis (88/90), the remaining two patients received peritoneal dialysis. While we started treatment at standard dose, other reports show 29 patients (48\%) started sunitinib therapy at a dose of $50 \mathrm{mg}$, 19 patients (31\%) at $37.5 \mathrm{mg}$ and $12(19 \%)$ at $25 \mathrm{mg}$. During therapy doses were augmented in six patients receiving $25 \mathrm{mg}$ and in one patient receiving $37.5 \mathrm{mg}$. One patient receiving $50 \mathrm{mg}$ and one receiving $37.5 \mathrm{mg}$ had their sunitinib doses reduced as a result of AEs. RECIST criteria of PD was met in six patients, $\mathrm{SD}$ - in nine, $\mathrm{PR}-$ in seven, and of CR in two. Some reports did not indicate response. Most common grade 3 events were hypertension and fatigue. Total number of grade 3 adverse events was 15 . Four patients had therapy withdraw: two due to intracranial hemorrhage, one due to cardiotoxicity and one due to fatigue. In relatively large (14 patients) analysis by Shetty et al. outcomes in patients with ESRD were similar to those in patients with normal kidney function [11]. In this analysis group of patients received variable treatment, with median number of treatment lines of 3 (range: 1-5) and with median total time of treatment of 27 months for sequential TKI/mTORi treatment and 28 months for TKI/mTORi and immunotherapy (IL-2 and INF- $\alpha$ ) treatment. This analysis reported PFS of targeted therapies including various lines of therapies and can't be used to compare efficacy of targeted therapies. In first-line treatment four patients received sunitinib, three - sorafenib, four - pazopanib, two - temsirolimus, one - bevacizumab [11]. In an Italian large study, median treatment duration (despite variable doses) for first-line sunitinib treatment was 12.7 months (1.4-27.2) and for sorafenib - 13.9 (2.7-40.1). Among patients treated with sunitinib 7/16 PR had and 5/15 SD and OS was not reached at data cut-off [10]. The presented summary highlights successful treatment of metastatic RCC using sunitinib in patients undergoing dialysis. Data both from our cohort and literature data support the statement that sunitinib and other TKI treatment in dialyzed patients suffering from $\mathrm{mRCC}$ is beneficial. However, calculated median PFS time may be slightly lower than PFS of nondialyzed patients [2] - a difference likely caused by the fact that in literature data PFS was not achieved in a 12 patients and general condition including comorbidities of these patients was not as good as in the case of patients without HD treatment. Data for studies treated with sorafenib are similar (Table 4) [10,35].

At this point of time PFS data are not available for homogenous population, since some of reports show 1st line data - TKI treatment [32], but other present analyses of patients treated previously with IFN or IL-2. Moreover, data for particular TKI - sunitinib, sorafenib, pazopanib - are also available for different lines, some patients are first-line TKI, but in others sequential treatment is presented. PFS from different lines of treatment and different sequences of treatment is noncomparable [11,45]. Moreover, differential starting dose and subsequent dosing with dose escalation and dose reduction schemes of particular TKI would not let to compare 
RESEARCH ARTICLE Czarnecka, Kawecki, Lian, Korniluk \& Szczylik

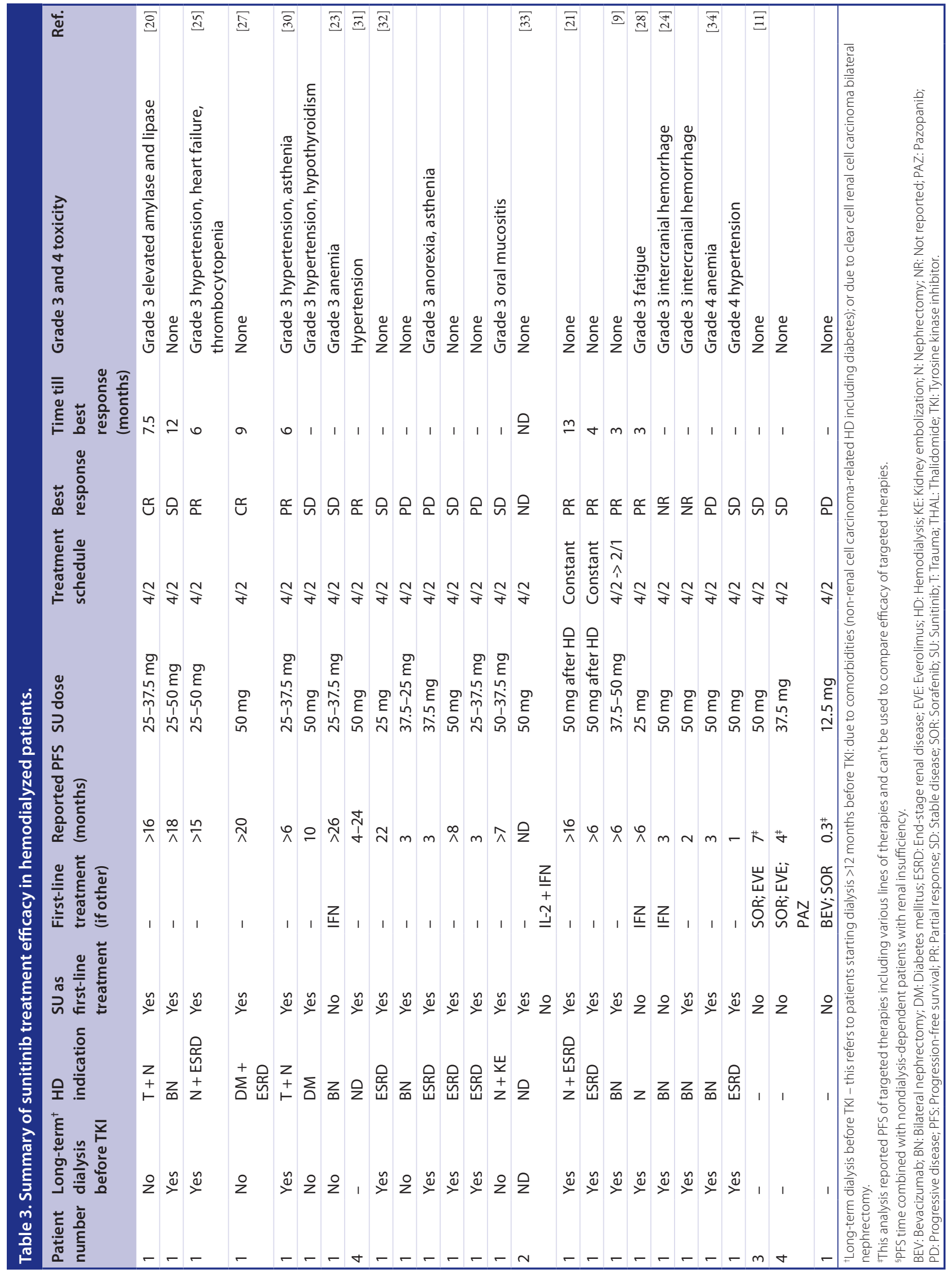




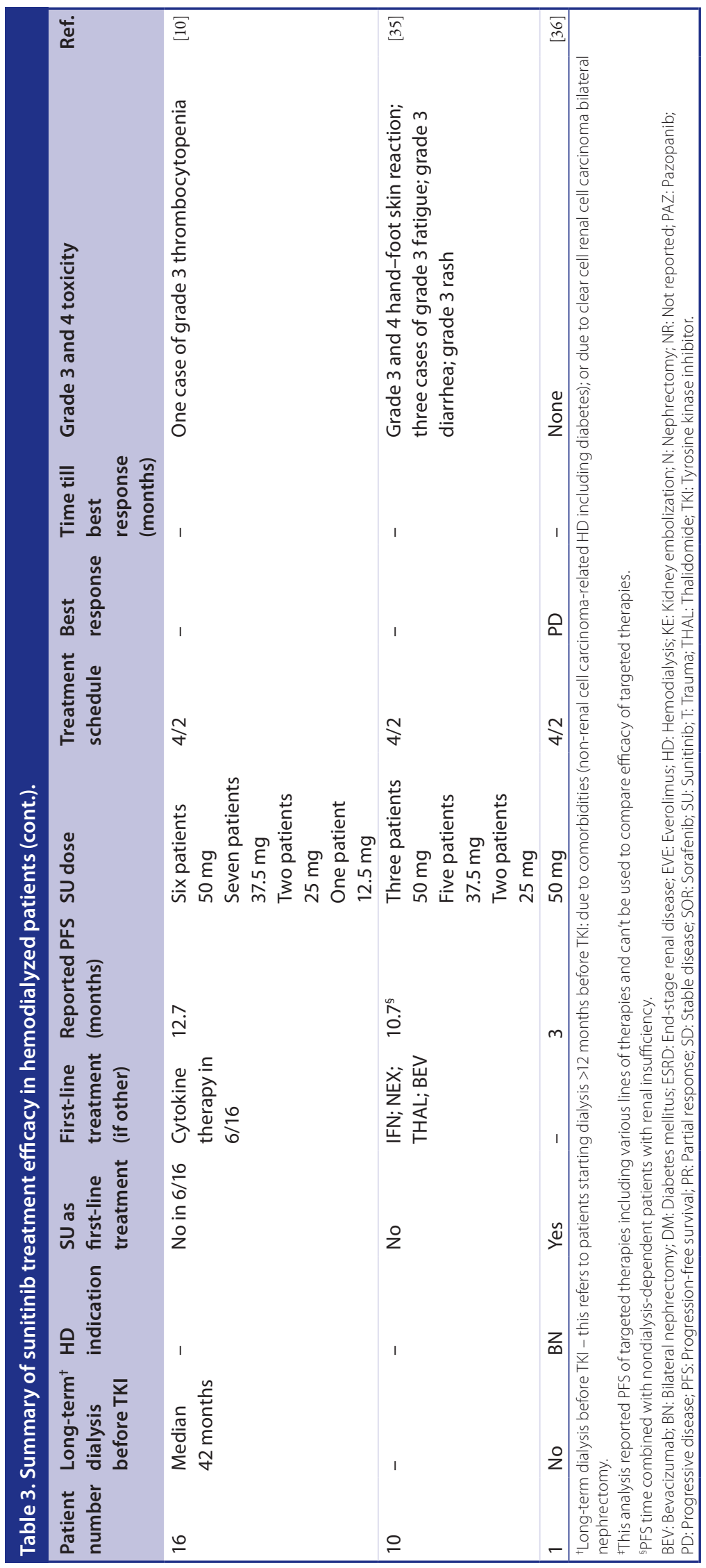


RESEARCH ARTICLE Czarnecka, Kawecki, Lian, Korniluk \& Szczylik

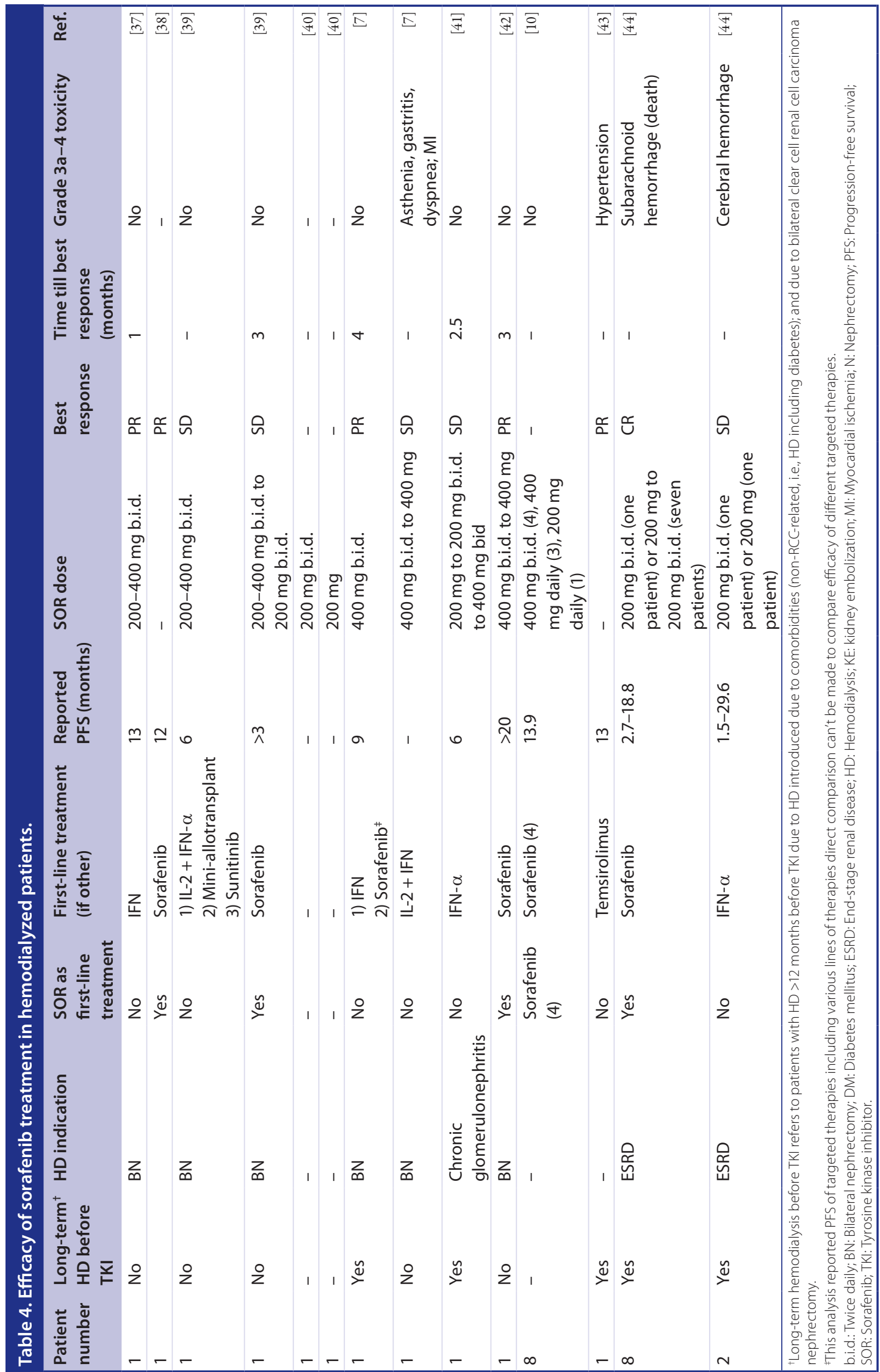


PFS data $[10-11,32]$. As reported the decision to start treatment with reduced doses of TKI was taken by the treating physicians due to lack of data on the use of TKIs in patients undergoing chronic dialysis, at the time of treatment decision [10]. It was suggested that there may not be significant difference in outcomes (PFS and OS) among patients who started TKIs on reduced doses and those who received full doses in HD patients [15], but this stays in contradiction to known correlation of TKI dose-intensity and efficacy [46-48]. Stable sunitinib serum levels were shown to be required to ensure continued disease control. Patients with the highest exposure to sunitinib had longer PFS and OS; as well as a higher probability of a response and a greater decrease in tumor size. Nevertheless, one need to remember that dose-intensity may be significantly influenced by intrapatient heterogeneity in metabolism and genetic background [47,49-51]. In particular, dose could be adjusted based on individual serum monitoring [52-55] or genetic profile [56], as in the case of axitinib [57,58].

\section{- Pharmacokinetics of RCC treatment in hemodialyzed patients}

Finally major RCC targeted therapies are multiple TKIs and growing evidence accumulate on their activity in patients with ESRD. Sorafenib is primarily metabolized in the liver by CYP3A4 and by UGT1A9 glucuronosyltransferase $[10,39]$. As a result, $77 \%$ of the total dose of sorafenib may be recovered from the feces, with $50 \%$ as unchanged drug and $17 \%$ as pyridine-N-oxide [40]. Only 19-20\% of the drug is excreted in the urine, mostly as glucuronide conjugates [10], which was confirmed in patients undergoing hemodialysis [40]. As a consequence of its limited renal clearance sorafenib appeared suitable for patients with mRCC and ESRD [10]. Sorafenib clearance is limited by its binding to plasma proteins including albumin, $\alpha$ - and $\beta$-globulin and LDL at a rate of $99.5 \%$ [41]. On the other hand, due to hypoalbuminemia frequent in ESRD [59], protein-unbound fraction of sorafenib may be higher in hemodialized patients and this may potentially relate to AEs [44]. Nevertheless, the elimination curve of sorafenib (at $400 \mathrm{mg}$ daily dose) in patients with severe renal function impairment is similar to that described in patients with mild renal insufficiency [60], and normal renal function $-\mathrm{C}_{\max }$, $\mathrm{t}_{1 / 2}$ and AUC [41,61]. At the same time studies of mRCC patients on hemodialysis revealed no accumulation of sorafenib after daily dosing at 200 mg daily. Plasma sorafenib and its active metabolite ( $\mathrm{N}$-oxide) concentrations did increase is hemodialized subjects $[15,40]$. Sorafenib and its active metabolite concentrations increased proceeding dialysis, and continuously reaching $\mathrm{C}_{\max }$ two to fourfold higher than obtained without hemodialysis [40-41,44], but it was hypothesized to be influenced by ultrafiltration of water (during dialysis) [41]. This hypothesis was rejected by Kennoki et al., who claimed that 5\% reduction of body fluid could not result in doubling of sorafenib plasma concentration increase. These authors stated that unique dynamic pattern of sorafenib concentration remains of undetermined clinical explanation and implication [44]. In Japanese study mean plasma concentration and AUC were similar both on the day of hemodialysis and off-hemodialysis [44]. At least four Phase I trials have addressed the problem of sorafenib steady-state and AUC in patients with renal impairment of different degrees and have proven no significant correlation [62-65]. Moreover, CALGB 60301 Phase I trial pharmacokinetic and Phase I study of sorafenib in patients with hepatic or renal dysfunction the authors recommended full-dose sorafenib - 400 mg b.i.d. for patients with mild renal insufficiency (59 $\mathrm{ml} / \mathrm{min} \geq \mathrm{CrCl} \geq 40 \mathrm{ml} / \mathrm{min}$ ) and doses reduction to $200 \mathrm{mg}$ b.i.d. for patients with moderate disease $(39 \mathrm{ml} / \mathrm{min} \geq \mathrm{CrCl} \geq 20 \mathrm{ml} / \mathrm{min}$ ) and $200 \mathrm{mg}$ once daily for patients on dialysis. No recommendations were made for patients with severe renal impairment $(\mathrm{CrCl}<20 \mathrm{ml} / \mathrm{min})$ who were not on dialysis $[66,67]$. It was later defined that sorafenib (and sunitinib) can be safely administered provided that adequate monitoring of renal function is applied on treatment [8].

As in the case of sorafenib, studies suggested that pharmacokinetics of sunitinib in subjects with severe renal impairment is similar to those with normal renal function $[9,62]$. Sunitinib is primarily metabolized in the liver by CYP3A4 and only up to $16 \%$ of the drug is excreted in urine [68]. Sunitinib and its active metabolite are known to be eliminated in the feces in 80 to $85 \%[32,62,69]$. Dialysis was reported not to affect sunitinib plasma concentration, as the compound was shown nondialyzable. In patients with ESRD single sunitinib dose clearance by hemodialysis is at insignificant level of 0.01-0.02\% [70]. Moreover, another study of sunitinib in two hemodialyzed patients confirmed sunitnib and its metabolite 
- SU12662 (N-desethyl metabolite) - hemodialysis clearance at $0 \mathrm{ml} / \mathrm{min}[71]$. Pharmacokinetic studies analyzing sunitinib usage in hemodialysed patients reported mean concentration within its therapeutic range $(50-100 \mathrm{ng} / \mathrm{ml})$ and AUC similar to patients without renal insufficiency $(1.259$ vs $1.888 \mu \mathrm{g} . \mathrm{h} / \mathrm{ml}$, respectively) [71]. Minimal concentrations of both sunitnib and SU012662 in such populations was: $84.6 \mathrm{ng} / \mathrm{ml},>50 \mathrm{ng} / \mathrm{ml}$ and 12 $\mathrm{ng} / \mathrm{ml}$ in three analyzed patients [9,71]. In general sunitinib doses of $50 \mathrm{mg} /$ day led to plasmatic Cmin ranging from 50 to $100 \mathrm{ng} / \mathrm{ml}$ in patients undergoing $\mathrm{HD}[9]$. On the other hand according to Khosravan et al. [70], single $50 \mathrm{mg}$ dose of sunitnib results in lower maximum concentration of sunitinib in patients with ESRD requiring dialyses when compared with patients without renal insufficiency or with severe renal impairment (14 vs 24 vs $24 \mathrm{ng} / \mathrm{ml}$, respectively) [70]. Moreover, Khan et al. has shown that plasma exposure to sunitinib and SU012662 in patients receiving HD was lower than in patients with normal renal function. Nevertheless, arterial and venous pharmacokinetic concentration data indicated that sunitinib was not eliminated by HD [36]. Mechanisms of this effect reported by Khan et al. and Khosravan et al. remain elusive - as dialysis do not affect sunitinib concentration directly - possible explanations include impaired drug absorption; drug interactions; or lower patient compliance [70]. It seems that sunitinib concentration may vary between patients significantly and possible intercalating factors should be under special attention. Higher AUC is associated with increased time to tumor progression, overall survival, response probability, and tumor size reduction in patients with metastatic RCC. Highest exposure to sunitinib correlated with highest probability of tumor response. Tentative relationships occurs also between AUC and incidence, but not severity, of fatigue and lower absolute neutrophil count; also total drug concentration correlated with diastolic blood pressure increase during therapy [47]. Finally, some authors have advocated for pharmacological monitoring of drug concentration in patients with abnormal renal function $[69,72]$.

\section{- TKI treatment schedule in RCC hemodialyzed patients}

Most common and well established sunitinib dosing schedule is 4 weeks of sunitinib administration ('on' period) followed by 2 weeks wash-out period ('off' period). With this treatment regimen sunitinib was administered for patients with renal insufficiency and acceptable tolerance and effectiveness was reported [36,73]. Among patients reported until now, two were treated with a modified schedule: $50 \mathrm{mg}$ of sunitinib after each dialysis. Interestingly, this treatment modification resulted in partial response for over 16 months in one patient and stable disease for over 6 months in the other, without serious AEs [21]. Although other treatment schedules were reported for general population [46,74], the lack of sufficient data excludes any direct comparison of different schedules in hemodialyzed patients. An Italian study reported no difference between patients treated on reduced dose and those treated with full dose [62]. Moreover, it was suggested that since sunitinib is not dialyzable it may be administered any time before or after the hemodialysis [9].

\section{- TKI treatment safety in RCC hemodialyzed patients}

Some studies already suggested that in subjects with severe renal impairment sunitinib treatment is safe and well tolerated [9]. As metabolism of TKIs occurs in mostly the liver it is hypothesized that these drugs are safe in patients with ESRD [11] and our experience seems to support this notion. At the same time it is also likely that renal impairment being associated with age and comorbidities including hypertension may lead to increased incidence or severity of TKI toxicities [65]. Most AEs reported in patients undergoing $\mathrm{HD}$ and TKI treatment were grade 1 or 2; and among those anemia was the most commonly reported [10]. In our report, and in Italian study, no patient had to change (increase) the number of dialysis sessions during TKI treatment [10].

In the analysis of patients with renal insufficiency with $\mathrm{CrCl}<60 \mathrm{ml}$ - the duration of sorafenib toxicities was longer and there was trend towards more grade 2 and 3 toxicities. In this study of 14 cases with $\mathrm{CrCl}<60 \mathrm{ml} / \mathrm{min}$ diarrhea was reported more frequent when compared with subjects with normal renal function of $\mathrm{CrCl}$ $>60 \mathrm{ml} / \mathrm{min}$ treated in the same ward -57 versus $33 \%$. Similarly HFSR was more often in those with renal insufficiency $-86 \%$ versus 56\% [65]. As a consequence, more dose modifications and prolonged dose interruptions were reported, but with no influence on PFS/OS [65]. At the same time no association between $\mathrm{OR}$ and occurrence of $\mathrm{AE}$ and pharmacokinetic parameters $\left(\mathrm{C}_{\max }\right.$ $\mathrm{C}_{\text {min }}, \mathrm{AUC}$ ) was reported [44]. In the Italian study grade 3 treatment-related AEs were observed only in patients treated with sorafenib and included 
nausea, fatigue and diarrhea. Moreover, one patient in this study discontinued treatment as a result of grade 3 cardiac $\mathrm{AE}$ (symptomatic cardiac ischemia) [10]. In another study of patients with $\mathrm{CrCl}<60 \mathrm{ml} / \mathrm{min}$ dose reduction was reported at $43 \%$ and treatment interruptions at $57 \%$, mainly due to hypertension and HFSR. At the same time in patients with $\mathrm{CrCl}>60 \mathrm{ml} / \mathrm{min}$ dose reduction was noted in $22 \%$ and treatment break in 28\% [65]. Moreover, in Japanese study of long-term dialyzed patients - with dialysis duration from 12 to 379 months prior to sorafenib treatment - grade 5 subarachnoid hemorrhage and grade 4 cerebellar hemorrhage were reported. There events were later show as related to the general condition of those patients [44]. Therefore, while starting TKI treatment, we need to especially consider the general condition of long-term hemodialyzed patients including cardiovascular comorbidities, including cardiac dysfunction, severe systemic atherosclerosis and bleeding tendency. Among ESRD patients, especially those on on long-term hemodialysis ( $>5$ years) should be treated with more careful surveillance due to severe AEs are likely to be common in this population as shown by the Japanese study. Moreover, surveillance in terms of blood monitoring is required, since hemodialysis patients are at high risk of platelet dysfunction due to anticoagulants used in hemodialysis and anemia and hypertension are often found in this population [44,62]. Procedure of hemodialysis involves usage of heparin, an agent linked to higher risk of developing internal hemorrhage, which may influence bleeding tendency. Although correlation between hemodialysis during sunitinib treatment and intracranial hemorrhage might be incidental, clinicians supervising such patients should be aware of potential risk. Further studies are needed to establish guidelines for the safe treatment of patients under hemodialysis [39]. Increase in cardiac toxicities observed in sorafenib patients has also been associated with long-term HD periods that is known to be related to high prevalence of impaired cardiac function, uncontrolled hypertension and atherosclerosis [55].

\section{Conclusion}

Sorafenib, sunitinib, pazopanib and axitinib registration trials included patients with normal kidney function [1-4]. However, it's estimated that chronic kidney disease might occur in up to $37 \%$ of patients with metastatic RCC and patients with ESRD may represent up to $2 \%$
ccRCC patients. Studies analyzing patients with $\mathrm{mRCC}$ and renal insufficiency (GFR $<60 \mathrm{ml} / \mathrm{min} \times 1.73^{-2}$ but without a need for dialysis) have shown that that efficacy and toxicity of sunitinib treatment in such patients are comparable to outcomes in patients without impaired renal function. Most common AEs described in such patient population is similar to those reported among patients without renal failure: fatigue, hand-foot syndrome, hypertension, neutropenia and diarrhea. At the same time chronic renal failure seemed not to alter sunitinib pharmacokinetics and pharmacodynamics $[36,73,75]$. TKI was show not to be contraindicated in patients with $\mathrm{mRCC}$ and ESRD undergoing $\mathrm{HD}$ [10].

Treatment with sunitinib and sorafenib is feasible in patient with ESRD with satisfactory efficacy and no unexpected toxicity. Results of treatment are similar to those in general $\mathrm{mRCC}$ population [10]. Our present study and profound literature analysis support the conclusion that efficacy and safety of TKI therapy in dialyzed patients is acceptable. Our analysis confirm the beneficial effect of TKI treatment of RCC patients on dialysis. The activity of sunitinib and sorafenib that we have observed in presented series of patients is alike that observed in Phase III trials (normal renal function). At the same time we aim to underline that patients with ESRD of any etiology and dialyzed patients have increased risk of developing RCC and the incidence of RCC is higher in all patients with ESRD [76]. Notably 1-2\% of patients on HD (or peritoneal dialysis) will develop RCC [65]. At the same time in long-term ( $>5$ years) HD-patients RCC biology is different, and the pathological features and clinical course is often more favorable [11]. The main histological types of RCCs arising in ESRD include: acquired cystic disease (ACD)-associated RCC; clear cell-papillary RCC; mucinous tubular and spindle-cell carcinoma (MTSCC); and Xp11.2 translocation/TFE3 gene fusion RCC [77]. In this group of patients other therapies might also be considered due to different genetic background of the disease, and among currently available mTOR inhibitor should be considered [78]. All together we conclude that undergoing dialysis should not limit access to effective metastatic RCC therapies. Nevertheless, due to qualitative and quantitative limitations of this and other studies, there is a demand for more profound (multicenter) research especially in the field of 
safety and QoL. Additional attention should be attributed to possibility of different severity of AEs if compared with in nondialyzed patients.

Maintaining proper exposure to sunitinib and its active metabolite SU012662 during therapy is a necessity for achieving therapeutic success in terms or ORR, PFS and OS. At this point of time dialysis seems not to interfere with AUC and mean concentration of sunitinib - therefore, from pharmacodynamics or pharmacokinetics perspective treatment of dialyzed patients with sunitinib, or sorafenib is more than justified. Similar data is needed for other TKIs including pazopanib and axitinib. Nevertheless, consensus or clinical guidelines have not been established for TKI administration in dialyzed patients [32].

\section{- Treatment guidelines}

Patients undergoing hemodialysis because of bilateral nephrectomy belong to a cohort that represents therapeutic challenges to clinicians. At this point of time there are no established guidelines on management of such patients by ESMO or NCCN. Therapy selection, administration and toxicity management in mRCC patients undergoing dialysis should be performed with caution and increased surveillance of AEs [24]. Careful dose escalation based on patients' tolerance may be recommended [41]. Currently, multiple reports support the possibility of regular dosing of sunitinib in patients treated with HD (standard dose and schedule). Clinical data about efficacy and safety support the statement that dialyzed patients suffering from metastatic RCC benefit from sunitinib or sorafenib therapy. Renal excretion of TKIs and their metabolites is low, so administration of these drugs may take place anytime, regardless of HD timing [36,44] and sunitinib or sorafenib may be administered before or after HD procedure, on the day of HD. Data available for different novel drugs used in metastatic RCC therapy sorafenib, everolimus and temsirolimus - suggest acceptable efficacy and safety when administered to hemodialyzed patients [44,79-80]. It should be remembered that clinicians may address dialyzed patients' needs with wide spectrum of therapies as first-line or second-line treatment.

\section{Future perspective}

New targeted drugs - TKIs including sunitinib, sorafenib, pazopanib and axitinib - are currently recommended as first- and/or second-line treatment in patients with ccRCC current data should be supplemented with studies addressing administration protocols (dose and schedule) and toxicity (AEs) surveillance in RCC patients with end-stage renal disease. Such data is required to optimize our current treatment strategies in specific patients population [24]. Prospective expanded access clinical trials, with pharmacokinetic studies, in a larger cohort of patients is required $[10,27]$.

Moreover, diabetic nephropathy is currently the most common cause of ESRD. Luckily some TKIs, including sunitinib have antihyperglycemic effects. Although the mechanism is not clear at this point of time [81]. On the contrary mTOR inhibitors - that are considered as standard in second/third-line treatment - may impair glucose tolerability and cause diabetic complications, including neuropathy and retinopathy. This problem and selection between TKI and mTOR inhibitor in treatment of patients with RCC and ESRD in second and subsequent lines needs to be addressed. The potentially pronephropatic mechanism of mTOR inhibitors should be considered in development of new therapies and drug combinations for RCC in general [82].

Financial \& competing interests disclosure

This work was supported by the Military Institute of Medicine statutory founding. Treatment cost was covered by Polish National Health Fund (NFZ). C Szczylik received consulting and lecture honoraria from Pfizer, Bayer HealthCare, Astellas, GlaxoSmithKline and Novartis. AM Czarnecka received lecture honoraria from Pfizer, GlaxoSmithKline, Bayer HealthCare and Novartis. The authors have no other relevant affiliations or financial involvement with any organization or entity with a financial interest in or financial conflict with the subject matter or materials discussed in the manuscript apart from those disclosed.

No writing assistance was utilized in the production of this manuscript.

\section{Ethical conduct of research}

The authors state that they have obtained appropriate institutional review board approval and have followed the principles outlined in the Declaration of Helsinki for all human or animal experimental investigations. In addition, for investigations involving human subjects, informed consent has been obtained from the participants involved.

\footnotetext{
Open access

This work is licensed under the Creative Commons Attribution-NonCommercial 3.0 Unported License. To view a copy of this license, visit http://creativecommons.org/ licenses/by-nc-nd/3.0/
} 


\section{EXECUTIVE SUMMARY}

\section{Background}

- Concomitance of renal insufficiency and renal clear cell carcinoma occurs.

- Data about sunitinib and other tyrosine kinase inhibitors usage among dialyzed patients are limited.

- Chronic toxicities generated by sunitinib, such as hypertension and proteinuria, may attribute to renal function impairment.

\section{Results}

- Out of 464 patients treated with tyrosine kinase inhibitors, nine patients were treated with dialysis due to end-stage renal disease.

- Sunitinib was safely and effectively used in five hemodialyzed patients.

- Sorafenib was safely and effectively used in three hemodialyzed patients.

- Pazopanib was safely and effectively used in one hemodialyzed patients.

- IFN- $\alpha$ was safely and effectively used in two hemodialyzed patients.

\section{Conclusion}

- Sunitinib metabolism is independent from sufficient renal function.

- Sunitinib is well tolerated in patients on hemodialysis.

- Sunitinib treatment efficacy is comparable to that reported in patients with normal renal function.

- Sunitinib dose reduction is not necessary in patients with end-stage renal disease.

- 4-week on/2-week off schedule is acceptable in terms of efficacy and tolerability for dialyzed patient treated with sunitinib.

- Sorafenib dose may be reduced down to $200 \mathrm{mg}$ twice daily or daily for patients on hemodialysis.

- Bevacizumab pharmacokinetic parameters are similar in patients with normal renal function and in patients requiring hemodialysis.

- Everolimus pharmacokinetic parameters are not influenced by hemodialysis.

- Temsirolimus undergoes hepatic metabolism and dose adjustment is not needed in patients on hemodialysis.

- Interferon alpha has a primarily renal metabolism and require modified interval between injections in patients undergoing dialysis.

- Intravenous IL-2 therapy can be safely performed in hemodialysis patients without dose modification.

\section{References}

Papers of special note have been highlighted as:

- of interest; $\bullet$ of considerable interest

1 Escudier B, Eisen T, Stadler WM et al. Sorafenib in advanced clear-cell renal-cell carcinoma. N. Engl. J. Med. 356(2), 125-134 (2007).

2 Motzer RJ, Hutson TE, Tomczak P et al. Sunitinib versus interferon alfa in metastatic renal-cell carcinoma. N. Engl. J. Med. 356(2), 115-124 (2007).

3 Sternberg CN, Davis ID, Mardiak J et al. Pazopanib in locally advanced or metastatic renal cell carcinoma: results of a randomized Phase III trial. J. Clin. Oncol. 28(6), 1061-1068 (2011).
4 Rini BI, Escudier B, Tomczak P et al. Comparative effectiveness of axitinib versus sorafenib in advanced renal cell carcinoma (AXIS): a randomised Phase 3 trial. Lancet 378(9807), 1931-1939 (2012).

5 Escudier B, Pluzanska A, Koralewski P et al. Bevacizumab plus interferon alfa-2a for treatment of metastatic renal cell carcinoma: a randomised, double-blind Phase III trial. Lancet 370 (9605), 2103-2111 (2007).

6 Mao FJ, Rini BI. The ineligible patient: how to treat patients not included in clinical studies. World J. Urol. 32(1), 9-18 (2014).

7 Ferraris E, Di Cesare P, Lasagna A, Paglino C, Imarisio I, Porta C. Use of sorafenib in two metastatic renal cell cancer patients with end-stage renal impairment undergoing replacement hemodialysis. Tumori 95(4), 542-544 (2009).

8 Zustovich F, Lombardi G, Farina P. Treating patients with metastatic renal carcinoma: an escape from Phase III. Expert Rev. Anticancer Ther. 12(7), 919-927 (2012).

9 Thiery-Vuillemin A, Montange D, Kalbacher $\mathrm{E}$ et al. Impact of sunitinib pharmacokinetic monitoring in a patient with metastatic renal cell carcinoma undergoing hemodialysis. Ann. Oncol. 22(9), 2152-2154 (2011).

10 Masini C, Sabbatini R, Porta C et al. Use of tyrosine kinase inhibitors in patients with metastatic kidney cancer receiving 
haemodialysis: a retrospective Italian survey. BJU Int. 110(5), 692-698 (2012).

-• Population-based study describing the safety and efficacy of tyrosine kinase inhibitors in patients with metastatic renal cell carcinoma and end-stage renal disease requiring hemodialysis. Covers 24 patients undergoing hemodialysis between July 2006 and December 2010 and estimates median progression-free and overall survival periods of this cohort.

11 Shetty AV, Matrana MR, Atkinson BJ, Flaherty AL, Jonasch E, Tannir NM. Outcomes of patients with metastatic renal cell carcinoma and end-stage renal disease receiving dialysis and targeted therapies: a single institution experience. Clin. Genitourin Cancer 12(5), 348-353 (2014).

- Relatively large report on patients with metastatic renal cell carcinoma and end-stage renal disease treated at the University of Texas MD Anderson Cancer Center from 2002 to 2012. Patient characteristics including demographic, histology, treatment, adverse events, duration of treatment and overall survival are reported.

12 Sternberg CN, Hawkins RE, Wagstaff J et al. A randomised, double-blind Phase III study of pazopanib in patients with advanced and/or metastatic renal cell carcinoma: final overall survival results and safety update. Eur. J. Cancer 49(6), 1287-1296 (2013).

13 Czarnecka A, Sobczuk P, Bogusz K, Spychalska M, Szczylik C. Survival, safety and treatment response duration in "real world" patients with metastatic clear cell renal cancer - an update from clinical practice. $B J U$ Int. 112(s3), 1-17 (2013).

14 Escudier B, Eisen T, Stadler WM et al. Sorafenib for treatment of renal cell carcinoma: final efficacy and safety results of the Phase III treatment approaches in renal cancer global evaluation trial. J. Clin. Oncol. 27(20), 3312-3318 (2009).

15 Beck J, Procopio G, Bajetta E et al. Final results of the European Advanced Renal Cell Carcinoma Sorafenib (EU-ARCCS) expanded-access study: a large open-label study in diverse community settings. Ann. Oncol. 22(8), 1812-1823 (2011).

16 Szmit S, Langiewicz P, Zlnierek J et al. Hypertension as a predictive factor for survival outcomes in patients with metastatic renal cell carcinoma treated with sunitinib after progression on cytokines. Kidney Blood Press Res. 35(1), 18-25 (2012).

17 Szmit S, Zaborowska M, Wasko-Grabowska A et al. Cardiovascular comorbidities for prediction of progression-free survival in patients with metastatic renal cell carcinoma treated with sorafenib. Kidney Blood Press Res. 35(6), 468-476 (2012).

18 Czarnecka AM, Oborska S, Rzepecki P, Szczylik C. Development of chronic myeloid leukaemia in patients treated with anti-VEGF therapies for clear cell renal cell cancer. Future Oncol. 11(1), 17-26 (2014).

19 Gore ME, Szczylik C, Porta C et al. Safety and efficacy of sunitinib for metastatic renal-cell carcinoma: an expanded-access trial. Lancet Oncol. 10 (8), 757-763 (2009).

20 Zastrow S, Froehner M, Platzek I, Novotny V, Wirth MP. Treatment of metastatic renal cell cancer with sunitinib during chronic hemodialysis. Urology 73(4), 868-870 (2009).

21 Yoon SH, Kim KH, Choi J et al. Novel sunitinib strategy in metastatic renal cell carcinoma on hemodialysis: intermittent dose of sunitinib after hemodialysis. Cancer Res. Treat. 42(3), 180-184 (2010).

22 Vickers MM, Choueiri TK, Rogers M et al. Clinical outcome in metastatic renal cell carcinoma patients after failure of initial vascular endothelial growth factor-targeted therapy. Urology 76(2), 430-434 (2010).

23 Tapiawala SN, Bargman JM, Oreopoulos DG, Simons M. Prolonged use of the tyrosine kinase inhibitor in a peritoneal dialysis patient with metastatic renal cell carcinoma: possible beneficial effects on peritoneal membrane and peritonitis rates. Int. Urol. Nephrol. 41(2), 431-434 (2009).

24 Syrios J, Kechagias G, Tsavaris N. Treatment of patients with metastatic renal cell carcinoma undergoing hemodialysis: case report of two patients and short literature review. BMC Nephrol. 14(1), 84 (2013).

25 Reckova M, Kakalejcik M, Beniak J. Treatment of hemodialyzed patient with sunitinib. Ann. Oncol. 20(2), 392-393 (2009).

26 Al-Najjar F, Jarkowski A, 3rd. Treatment of concurrent metastatic renal cell carcinoma and chronic myelogenous leukemia - easier said than done? A case report. J. Oncol. Pharm. Pract. 17(4), 436-439 (2011).

27 Park CY. Successful sunitinib treatment of metastatic renal cell carcinoma in a patient with end stage renal disease on hemodialysis. Anticancer Drugs 20 (9), 848-849 (2009).

28 Noda S, Kageyama S, Tsuru T et al. Pharmacokinetic/pharmacodynamic analysis of a hemodialyzed patient treated with $25 \mathrm{mg}$ of sunitinib. Case Rep Oncol. 5(3), 627-632 (2012).

29 Stadler WM, Figlin RA, Mcdermott DF et al. Safety and efficacy results of the advanced renal cell carcinoma sorafenib expanded access program in North America. Cancer 116(5), 1272-1280 (2010).

30 Vickers MM, Heng DY, Hemmelgarn B, Eigl BJ. Tolerance of sunitinib in dialyzed patients with metastatic renal cell carcinoma. Clin. Genitourin Cancer 7(3), E104-E106 (2009).

31 Lainakis G, Bamias A, Psimenou E, Fountzilas G, Dimopoulos MA. Sunitinib treatment in patients with severe renal function impairment: a report of four cases by the Hellenic Cooperative Oncology Group. Clin. Nephrol. 72(1), 73-78 (2009).

32 Park S, Lee J, Park SH et al. Treatment of hemodialyzed patients with sunitinib in renal cell carcinoma. Chemotherapy 56(6), 485-491 (2010).

33 Izzedine H, Massard C, Spano JP, Goldwasser F, Khayat D, Soria JC. VEGF signalling inhibition-induced proteinuria: mechanisms, significance and management. Eur. J. Cancer 46(2), 439-448 (2010).

34 Yildiz I, Sen F, Kilic L, Ciftci R, Basaran M. Intolerance to sunitinib treatment in hemodialysis patients with metastatic renal cell carcinoma. Korean J. Urol. 55(1), 74-76 (2014).

35 Josephs D, Hutson TE, Cowey CL et al. Efficacy and toxicity of sunitinib in patients with metastatic renal cell carcinoma with severe renal impairment or on haemodialysis. BJU Int. 108(8), 1279-1283 (2011).

36 Khan G, Golshayan A, Elson P et al. Sunitinib and sorafenib in metastatic renal cell carcinoma patients with renal insufficiency. Ann. Oncol. 21(8), 1618-1622 (2010).

37 Ruppin S, Protzel C, Klebingat KJ, Hakenberg OW. Successful sorafenib treatment for metastatic renal cell carcinoma in a case with chronic renal failure. Eur. Urol. 55(4), 986-988; quiz 988 (2009).

38 Thibault F, Izzedine H, Sultan V et al. Regression of vena cava tumour thrombus in response to sorafenib. Prog. Urol. 18(7), 480-482 (2008).

39 Rey PM, Villavicencio H. Sorafenib: tolerance in patients on chronic hemodialysis: a single-center experience. Oncology 74(3-4), 245-246 (2008).

40 Hilger RA, Richly H, Grubert $\mathrm{M}$ et al. Pharmacokinetics of sorafenib in patients with renal impairment undergoing hemodialysis. Int. J. Clin. Pharmacol. Ther. 47(1), 61-64 (2009).

41 Shinsako K, Mizuno T, Terada T et al. Tolerable sorafenib therapy for a renal cell carcinoma patient with hemodialysis: a case 
study. Int. J. Clin. Oncol. 15(5), 512-514 (2010).

42 Castagneto B, Stevani I, Giorcelli L et al. Sustained response following sorafenib therapy in an older adult patient with advanced renal cancer on hemodialysis: a case report. Med. Oncol. 28(4), 1384-1388 (2011).

43 Kato S, Shibamori K, Hiyama Y et al. Treatment of hemodialyzed patient by targeted therapy in metastatic renal cell carcinoma: a case report. Nihon Hinyokika Gakkai Zasshi. 104(4), 605-608 (2013).

44 Kennoki T, Kondo T, Kimata N et al. Clinical results and pharmacokinetics of sorafenib in chronic hemodialysis patients with metastatic renal cell carcinoma in a single center. Jpn J. Clin. Oncol. 41(5), 647-655 (2011).

- Interesting case series analysis describing the adverse events of tyrosine kinase inhibitor treatment in patients on long-term hemodialysis examining the influence of pharmacokinetic parameters to their benefit.

45 Gupta S, Parsa V, Heilbrun LK et al. Safety and efficacy of molecularly targeted agents in patients with metastatic kidney cancer with renal dysfunction. Anticancer Drugs 22(8), 794-800 (2011).

46 Atkinson BJ, Kalra S, Wang X et al. Clinical outcomes in patients with metastatic renal cell carcinoma treated with alternative sunitinib schedules. J. Urol. 191(3), 611-618 (2013).

47 Houk BE, Bello CL, Poland B, Rosen LS, Demetri GD, Motzer RJ. Relationship between exposure to sunitinib and efficacy and tolerability endpoints in patients with cancer: results of a pharmacokinetic/ pharmacodynamic meta-analysis. Cancer Chemother. Pharmacol. 66(2), 357-371 (2010).

48 Motzer RJ, Hutson TE, Olsen MR et al. Randomized Phase II trial of sunitinib on an intermittent versus continuous dosing schedule as first-line therapy for advanced renal cell carcinoma. J. Clin. Oncol. 30(12), 1371-1377 (2012).

49 Britten CD, Kabbinavar F, Hecht JR et al. A Phase I and pharmacokinetic study of sunitinib administered daily for 2 weeks, followed by a 1-week off period. Cancer Chemother. Pharmacol. 61(3), 515-524 (2008).

50 Houk BE, Bello CL, Kang D, Amantea M. A population pharmacokinetic meta-analysis of sunitinib malate (SU11248) and its primary metabolite (SU12662) in healthy volunteers and oncology patients. Clin. Cancer Res. 15(7), 2497-2506 (2009).
51 Mendel DB, Laird AD, Xin X et al. In vivo antitumor activity of SU11248, a novel tyrosine kinase inhibitor targeting vascular endothelial growth factor and platelet-derived growth factor receptors: determination of a pharmacokinetic/pharmacodynamic relationship. Clin. Cancer Res. 9(1), 327-337 (2003).

52 Schmidinger M, Arnold D, Szczylik C, Wagstaff J, Ravaud A. Optimizing the use of sunitinib in metastatic renal cell carcinoma: an update from clinical practice. Cancer Invest. 28(8), 856-864 (2011).

53 Di Lorenzo G, Porta C, Bellmunt J et al. Toxicities of targeted therapy and their management in kidney cancer. Eur. Urol. 59(4), 526-540 (2011).

54 Eisen T, Sternberg CN, Robert C et al. Targeted therapies for renal cell carcinoma: review of adverse event management strategies. J. Natl Cancer Inst. 104(2), 93-113 (2012).

55 Minkin P, Zhao M, Chen Z, Ouwerkerk J, Gelderblom H, Baker SD. Quantification of sunitinib in human plasma by highperformance liquid chromatography-tandem mass spectrometry. J. Chromatogr. B Analyt Technol. Biomed. Life Sci. 874(1-2), 84-88 (2008).

56 Beuselinck B, Karadimou A, Lambrechts D et al. Single-nucleotide polymorphisms associated with outcome in metastatic renal cell carcinoma treated with sunitinib. $B r$. J. Cancer 108(4), 887-900 (2013).

57 Rini BI, Garrett M, Poland B et al. Axitinib in metastatic renal cell carcinoma: results of a pharmacokinetic and pharmacodynamic analysis. J. Clin. Pharmacol. 53(5), 491-504 (2013).

58 Rini BI, Melichar B, Ueda T et al. Axitinib with or without dose titration for first-line metastatic renal-cell carcinoma: a randomised double-blind Phase 2 trial. Lancet Oncol. 14(12), 1233-1242 (2013).

59 Haller C. Hypoalbuminemia in renal failure: pathogenesis and therapeutic considerations. Kidney Blood Press Res. 28(5-6), 307-310 (2005).

60 Kane RC, Farrell AT, Saber H et al. Sorafenib for the treatment of advanced renal cell carcinoma. Clin. Cancer Res. 12(24), 7271-7278 (2006).

61 Smith W, Kipnes MS, Marbury TC et al. Effects of renal impairment on the pharmacokinetics and safety of sorafenib. EJC Supplements 7(2), 434 (2009).

62 Strumberg D, Richly H, Hilger RA et al. Phase I clinical and pharmacokinetic study of the novel Raf kinase and vascular endothelial growth factor receptor inhibitor BAY 43-9006 in patients with advanced refractory solid tumors. J. Clin. Oncol. 23(5), 965-972 (2005).

63 Clark JW, Eder JP, Ryan D, Lathia C, Lenz HJ. Safety and pharmacokinetics of the dual action Raf kinase and vascular endothelial growth factor receptor inhibitor, BAY 43-9006, in patients with advanced, refractory solid tumors. Clin. Cancer Res. 11(15), 5472-5480 (2005).

64 Moore M, Hirte HW, Siu L et al. Phase I study to determine the safety and pharmacokinetics of the novel Raf kinase and VEGFR inhibitor BAY 43-9006, administered for 28 days on $/ 7$ days off in patients with advanced, refractory solid tumors. Ann. Oncol. 16(10), 1688-1694 (2005).

65 Parsa V, Heilbrun L, Smith D, Sethi A, Vaishampayan U. Safety and efficacy of sorafenib therapy in patients with metastatic kidney cancer with impaired renal function. Clin. Genitourin Cancer 7(2), E10-E15 (2009).

66 Miller AA, Murry DJ, Owzar K et al. Phase I and pharmacokinetic study of sorafenib in patients with hepatic or renal dysfunction: CALGB 60301. J. Clin. Oncol. 27(11), 1800-1805 (2009).

67 Bellmunt J, Fishman M, Eisen T, Quinn D. Expert opinion on the use of first-line sorafenib in selected metastatic renal cell carcinoma patients. Expert Rev. Anticancer Ther. 10(6), 825-835 (2010).

68 Miller DC, Schonlau M, Litwin MS, Lai J, Saigal CS. Renal and cardiovascular morbidity after partial or radical nephrectomy. Cancer 112(3), 511-520 (2008).

69 Faivre S, Delbaldo C, Vera K et al. Safety, pharmacokinetic, and antitumor activity of SU11248, a novel oral multitarget tyrosine kinase inhibitor, in patients with cancer. J. Clin. Oncol. 24(1), 25-35 (2006).

70 Khosravan R, Toh M, Garrett M et al. Pharmacokinetics and safety of sunitinib malate in subjects with impaired renal function. J. Clin. Pharmacol. 50(4), 472-481 (2010).

71 Izzedine H, Etienne-Grimaldi MC, Renée N, Vignot S, Milano G. Pharmacokinetics of sunitinib in hemodialysis. Ann. Oncol. 20(1), 190-192 (2009).

72 Motzer RJ, Michaelson MD, Redman BG et al. Activity of SU11248, a multitargeted inhibitor of vascular endothelial growth factor receptor and platelet-derived growth factor 
receptor, in patients with metastatic renal cell carcinoma. J. Clin. Oncol. 24(1), 16-24 (2006).

73 Kim KH, Kim HY, Kim HR et al. Efficacy and toxicity of sunitinib in patients with metastatic renal cell carcinoma with renal insufficiency. Eur. J. Cancer 50(4), 746-752 (2014).

74 Barrios $\mathrm{CH}$, Hernandez-Barajas D, Brown MP et al. Phase II trial of continuous once-daily dosing of sunitinib as first-line treatment in patients with metastatic renal cell carcinoma. Cancer 118(5), 1252-1259 (2012).

75 Macfarlane R, Heng DY, Xie W et al. The impact of kidney function on the outcome of metastatic renal cell carcinoma patients treated with vascular endothelial growth factor-targeted therapy. Cancer 118(2), 365-370 (2012).

76 Farivar-Mohseni H, Perlmutter AE, Wilson S, Shingleton WB, Bigler SA, Fowler JE Jr. Renal cell carcinoma and end stage renal disease. J. Urol. 175(6), 2018-2020; discussion 2021 (2006).

77 Nouh MA, Kuroda N, Yamashita M et al. Renal cell carcinoma in patients with end-stage renal disease: relationship between histological type and duration of dialysis. BJU Int. 105(5), 620-627 (2010).

78 Voss MH, Bastos DA, Karlo CA et al. Treatment outcome with mTOR inhibitors for metastatic renal cell carcinoma with nonclear and sarcomatoid histologies. Ann. Oncol. 25(3), 663-668 (2014).
79 Thiery-Vuillemin A, Curtit E, Maurina T et al. Hemodialysis does not affect everolimus pharmacokinetics: two cases of patients with metastatic renal cell cancer. Ann. Oncol. 23(11), 2992-2993 (2012).

80 Miyake H, Harada KI, Kusuda Y, Fujisawa M. Efficacy and safety of temsirolimus in Japanese patients with metastatic renal cell carcinoma on hemodialysis. Int. J. Clin. Oncol. 18(6), 1054-1059 (2012).

81 Prada PO, Saad MJ. Tyrosine kinase inhibitors as novel drugs for the treatment of diabetes. Expert Opin. Investig. Drugs 22(6), 751-763 (2013).

82 Czarnecka AM, Kornakiewicz A, Lian F, Szczylik C. Future perspectives for mTOR inhibitors in renal cell cancer treatment. Future Oncol. 11(5), 801-817 (2015). 